\title{
Pravastatin-induced improvement in coronary reactivity and circulating ATP and ADP levels in young adults with type 1 diabetes
}

\author{
Tuomas O. Kiviniemi ${ }^{1,2 *+}$, Gennady G. Yegutkin ${ }^{3 \dagger}$, Jyri O. Toikka ${ }^{1}$, Subhadeep Paul' ${ }^{4}$, Tero Aittokallio ${ }^{5}$, \\ Tuula Janatuinen ${ }^{1,6}$, Juhani Knuuti ${ }^{6}$, Tapani Rönnemaa ${ }^{2}$, Juha W. Koskenvuo ${ }^{1,6,7}$, Jaakko J. Hartiala ${ }^{1}$, \\ Sirpa Jalkanen ${ }^{3}$ and Olli T. Raitakari ${ }^{1,7}$
}

\author{
' Department of Clinical Physiology, Turku University Hospital, Turku, Finland \\ ${ }^{2}$ Department of Medicine, Turku University Hospital, Turku, Finland \\ ${ }^{3}$ Medicity Research Laboratory, University of Turku, Turku, Finland \\ ${ }^{4}$ Department of Mathematics, IIT Kharagpur, India \\ ${ }_{5}^{5}$ Institute for Molecular Medicine, University of Helsinki, Helsinki, Finland \\ ${ }^{6}$ Turku PET Centre, Turku University Hospital, Turku, Finland \\ ${ }^{7}$ Research Centre of Applied and Preventive Cardiovascular Medicine, University of Turku, Turku, Finland
}

Edited by:

Mikko P. Tulppo, Verve, Finland

Reviewed by:

Arkady Rutkovskiy, University of

Oslo, Norway

Shiang Y. Lim, University of

Melbourne, Australia

\section{*Correspondence:}

Tuomas O. Kiviniemi, Department of Medicine, Turku University Hospital, Kiinanmyllynkatu 4-8,

FIN-20520 Turku, Finland.

e-mail: tuoski@utu.fi

these authors contributed equally to this work.

\begin{abstract}
Aims: Extracellular ATP and ADP regulate diverse inflammatory, prothrombotic and vasoactive responses in the vasculature. Statins have been shown to modulate their signaling pathways in vitro. We hypothesized that altered intravascular nucleotide turnover modulates vasodilation in patients with type 1 diabetes (T1DM), and this can be partly restored with pravastatin therapy. Methods: In this randomized double blind study, plasma ATP and ADP levels and echocardiography-derived coronary flow velocity response to cold pressor test (CPT) were concurrently assessed in 42 normocholesterolemic patients with T1DM (age $30 \pm 6$ years, LDL cholesterol $2.5 \pm 0.6 \mathrm{mmol} / \mathrm{L}$ ) before and after four-month treatment with pravastatin $40 \mathrm{mg} /$ day or placebo $(n=22$ and $n=20$, respectively), and in 41 healthy control subjects. Results: Compared to controls, T1DM patients had significantly higher concentrations of ATP $(p<0.01)$ and ADP $(p<0.01)$ and these levels were partly restored after treatment with pravastatin $(p=0.002$ and $p=$ 0.007 , respectively), but not after placebo ( $p=0.06$ and $p=0.14$, respectively). Coronary flow velocity acceleration was significantly lower in T1DM patients compared to control subjects, and it increased from pre- to post-intervention in the pravastatin $(p=0.02)$, but not in placebo group $(p=0.15)$. Conclusions: Pravastatin treatment significantly reduces circulating ATP and ADP levels of T1DM patients, and concurrently improves coronary flow response to $\mathrm{CPT}$. This study provides a novel insight in purinergic mechanisms involved in pleiotropic effects of pravastatin.
\end{abstract}

Keywords: ATP, ADP, soluble nucleotidases, coronary flow, pleiotropic effect

\section{INTRODUCTION}

Extracellular ATP and ADP are important signaling molecules regulating diverse signaling responses in cardiovascular, nervous and other systems (Ralevic and Burnstock, 1998; Bours et al., 2006; Erlinge and Burnstock, 2008). Released into extracellular fluids by exocytosis from nucleotide-containing granules, by efflux through a membrane transport system in response to cell activation, or as a consequence of cell death, they act as paracrine or autocrine mediators of inflammation (Bours et al., 2006). Moreover, they cause vasodilation via selective activation of a series of G-protein-coupled (P2Y) or ligandgated (P2X) receptors (Ralevic and Burnstock, 1998; Erlinge and Burnstock, 2008; Mercier et al., 2012). ATP may stimulate vascular smooth muscle cell (VSMC) growth, migration, release of matrix metalloproteinases and osteopontin that may contribute to the development of diabetic microvascular disease (Erlinge and Burnstock, 2008). Abnormal reactive hyperemia as assessed with flow-mediated dilation (FMD) of brachial artery (Järvisalo et al., 2004) or abnormal coronary microcirculatory reactivity (Pitkänen et al., 1998) exists in patients with type 1 diabetes (T1DM) before any clinical signs or symptoms of macrovascular disease. The role of purinergic signaling cascade in the regulation of arterial vasodilation in the early stages of atherosclerosis of patients T1DM, however, remains largely unknown.

Statins activate the phosphoinositol-3 kinase and Akt signaling pathway, and in addition, increase expression and activity of endothelial enzymes such as nitric oxide synthase, cyclooxygenase-2 and ecto-5' -nucleotidase (Laufs et al., 1998; Ledoux et al., 2002; Merla et al., 2007). Ecto-5'-nucleotidase mediates rapid dephosphorylation of ATP/ADP-derived AMP further to adenosine, which has vasodilatory, anti-inflammatory (Bours et al., 2006) as well as cardioprotective responses against hypoxia (Eltzschig et al., 2003). The ability of atorvastatin to 
up-regulate ATPase, ADPase and ecto-5' -nucleotidase (AMPase) activities in human valve interstitial cells (Osman et al., 2006) suggests that the whole purinergic cascade could be modulated under the action of statins.

The aims of this trial were (1) to perform comprehensive comparative analysis of circulating ATP and ADP levels, soluble nucleotidase activities, coronary flow responses to $\mathrm{CPT}$ in control healthy subjects vs young asymptomatic, normocholesterolemic patients with non-complicated T1DM, and (2) to assess whether these biochemical and hemodynamic parameters would be concurrently modulated in T1DM patients in a prospective randomized placebo controlled four-month treatment with pravastatin.

\section{MATERIALS AND METHODS PATIENT SELECTION AND STUDY DESIGN}

Forty-two patients with non-complicated T1DM participated in the study. The inclusion criteria were: age 18-40 years, diabetes duration 3-25 years, no symptoms or diagnosis of cardiovascular disease or asthma, no use of cardiovascular medication or antioxidants, no diagnosis of proliferative retinopathy, FMD of the brachial artery less than $10 \%, \mathrm{HbA}_{1 \mathrm{c}}$ less than $10 \%$, LDL cholesterol less than $4.0 \mathrm{mmol} / \mathrm{L}$ and normal liver function. Altogether 57 patients were screened for the study. The patients were recruited by advertisement and from diabetes clinics in Turku and surrounding areas. The patients were interviewed for medical history, alcohol and caffeine use, physical activity and family history of premature coronary artery disease. In addition, serum human chorionic gonadotropin level was measured from all female patients to exclude pregnancy. The subjects were randomized in a double blind manner to receive either pravastatin $40 \mathrm{mg} /$ day $(n=22)$ or placebo $(n=20)$ for four months. Bristol-Myers Squibb, Finland, provided Pravastatin $40 \mathrm{mg}$ tablets and matching placebos. In order to control for use of dietary fats, the use of bread spread was standardized by providing all subjects margarine (total fat content $60 \mathrm{~g} / 100 \mathrm{~g}$ whereof $18 \mathrm{~g}$ saturated, $29 \mathrm{~g}$ monounsaturated and $13 \mathrm{~g}$ polyunsaturated, vitamin $\mathrm{E}$ $12 \mathrm{mg} / 100 \mathrm{~g}$, vitamin A $900 \mu \mathrm{g} / 100 \mathrm{~g}$ and vitamin D $7.5 \mu \mathrm{g} / 100 \mathrm{~g}$ ) and advising to use $20 \mathrm{~g} /$ day of this product on bread. Otherwise the subjects were advised to adhere to their normal diet. The control group consisted of 41 healthy (mean age $24 \pm 2.3$ years) normocholesterolemic, non-hypertensive, and non-smoking Caucasian men.

The Local Ethical Committee of the Turku University Hospital approved the study protocol. The study was conducted according to the principles expressed in the Declaration of Helsinki. The study protocol and the potential risks of the study were explained in detail to the patients and thereafter a written informed consent was obtained.

Blood samples were taken after an overnight fast on study mornings from the antecubital vein. Blood was collected into tubes for preparing plasma and serum blood samples, respectively. For serum preparation, blood was allowed to clot before centrifugation (10 $\mathrm{min}$ at $1500 \mathrm{~g}$ ) while plasma samples (EDTA) were immediately centrifuged and freezed at $-80^{\circ} \mathrm{C}$. Serum lipid levels, HbAlc were determined as previously reported (Janatuinen et al., 2004).

\section{QUANTIFICATION OF ATP AND ADP LEVELS IN HUMAN PLASMA}

Plasma ATP and ADP were determined by enzyme-coupled assay using ATPlite assay kit with a long-lived luminescent signal (Perkin Elmer, Groningen, The Netherlands) as described elsewhere (Mercier et al., 2012). Briefly, 10- $\mu$ l aliquots of EDTA-plasma from healthy as well as placebo and pravastatintreated T1DM were transferred into two parallel wells of white non-phosphorescent 96-well microplate containing $100 \mu \mathrm{l}$ PBS with (A) or without (B) mixture of $200 \mu \mathrm{M}$ UTP and $5 \mathrm{U} / \mathrm{ml}$ of NDP kinase from baker's yeast S. cerevisiae (Sigma). Subsequent to addition of $50 \mu l$ ATP-monitoring reagent containing luciferin/luciferase mixture, luminescence of the samples was measured using Tecan Infinite M200 microplate reader (Salzburg, Austria). The differences in luminescence signals between well "A" (ATP + ADP) and "B" (only ATP) allowed quantifying the concentration of ADP, which was converted into ATP through the NDP kinase mediated reaction in the presence of exogenous UTP. Such approach allows simultaneous measurement of both ATP and ADP content within the same sample. Plasma haemoglobin concentration was also determined by measuring the absorbance at the peak of the Soret band $(415 \mathrm{~nm})$ and also at 380 and $450 \mathrm{~nm}$, as described previously (Mercier et al., 2012). Hemoglobin levels in all analyzed blood samples did not exceed $4.0 \mathrm{mg} / \mathrm{dL}$ (data not shown).

\section{MEASUREMENT OF SOLUBLE NUCLEOTIDASE ACTIVITIES}

Soluble NTPDase and 5'-nucleotidase activities were assayed radiochemically, as described earlier (Yegutkin et al., 2007). Specifically, for ADPase/NTPDase activity, serum (10 $\mu \mathrm{l})$ was incubated $60 \mathrm{~min}$ at $37^{\circ} \mathrm{C}$ in $80 \mu \mathrm{l}$ RPMI- 1640 medium containing $5 \mathrm{mM} \quad \beta$-glycerophosphate, $80 \mu \mathrm{M}$ of adenylate kinase inhibitor Ap5A and $50 \mu \mathrm{M}$ ADP with tracer [2,8-3H]ADP (Perkin Elmer, Boston, USA). Likewise, 5'nucleotidase activity was assayed by incubating $10 \mu \mathrm{l}$ serum for $60 \mathrm{~min}$ at $37^{\circ} \mathrm{C}$ in $80 \mu \mathrm{l}$ RPMI- 1640 with $5 \mathrm{mM} \beta$ glycerophosphate, $300 \mu \mathrm{M}$ [2-3H]AMP (Quotient Bioresearch, GE Healthcare, Rushden, UK). Aliquots of the mixture $(8 \mu \mathrm{l}$; $\sim 5 \times 10^{5} \mathrm{dpm} / \mathrm{spot}$ ) were applied to Alugram SIL G/UV 254 sheets (Macherey-Nagel, Duren, Germany) and separated by thin-layer chromatography using appropriate solvent mixture (Yegutkin and Burnstock, 1998). Radiolabeled substrates and their dephosphorylated products were quantified by scintillation $\beta$-counting and nucleotidase activities were expressed as nanomoles of ${ }^{3} \mathrm{H}$-substrate metabolized per hour by $1 \mathrm{ml}$ serum.

Physiological variability of circulating nucleotide levels and soluble nucleotidase activities were assessed in three males and three females. Blood samples were collected after overnight fasting week apart three times. Coefficients of variation were 0.47 , $0.47,0.18$, and 0.27 for ATP, ADP, NTPDase, and 5'-nucleotidase, respectively.

\section{ECHOCARDIOGRAPHY PROTOCOL}

Transthoracic echocardiography studies were performed with Sequoia C 512 ultrasound mainframe (Acuson Inc., Mountain View, California, USA) with a standard $3.5 \mathrm{MHz}$ transducer as previously described (Kiviniemi, 2008). B-mode and colorDoppler mapping were used to identify the distal LAD. 
The coronary flow velocity was assessed with a pulsed wave Doppler and mean diastolic flow velocities (MDV) were measured.

\section{COLD PRESSOR TEST}

The studies were performed after overnight fasting. Alcohol and caffeine were prohibited $12 \mathrm{~h}$ before the study. On the study morning, the subjects took one-half of their normal long-acting insulin and no short-acting insulin. Baseline values of coronary flow velocity were detected as an average of three separate flow velocity measurements. After the baseline measurements, the subjects' right hand was placed into ice-cold water for 120 s. During the hand immersion, flow velocity was measured continuously to allow time course sampling of MDV values as previously described (Kiviniemi et al., 2007). The hyperemia/baseline-ratio of MDV during CPT, the time to the peak MDV during CPT from $0 \mathrm{~s}$ time point and the average acceleration slope to the peak were measured as previously described (Dimitrow et al., 2000). The response profiles related to time to peak MDV and acceleration slope to the peak were based on mathematical imputation using non-parametric regression which can give reliable results for reasonable amount of available observations. Profiles with less than $20 \%$ data points available throughout the CPT have been discarded to improve the quality of findings. Therefore, adequate coronary flow velocity tracings could be obtained in 14 and 17 patients in pravastatin and placebo groups, respectively. This was due to patients were moving during cold pressor test (CPT) hand immersion.

CPT was generally well tolerated. Intra and inter-observer variabilities of the CPT measurement (hyperemia to baseline ratio) were coefficient of variation $(\mathrm{CV}) 3.0 \pm 2.0 \%$, and $3.7 \pm 3.7 \%$ respectively, and mean difference $0.0(-0.1$ to 0.1$)$ and $0.0(-0.2$ to 0.2 ), respectively.

\section{STATISTICAL ANALYSES}

Results are presented as mean value \pm SD unless stated otherwise. Primary prospectively determined endpoint of CPT response included hyperemia to baseline ratio, and co-primary end points the time to the peak MDV during CPT from $0 \mathrm{~s}$ time point, and the average acceleration slope to the peak (Dimitrow et al., 2000). Sample size of 16 subjects was calculated for each group with a known SD of 0.5 for CPT hyperemia to baseline ratio and an assumed difference of 0.5 between the interventions $(\alpha=0.05, \beta=0.80)$. ATP, ADP levels, and soluble NTPDase and $5^{\prime}$-nucleotidase activity determinations and their association with CPT values were assessed on a post hoc basis. Comparisons between the groups were analyzed using the non-parametric Mann-Whitney $U$-test, sign test and Wilcoxon signed-rank tests, or using the parametric unpaired Student's $t$-test where appropriate. For comparison between pre- and post-intervention levels in the same group, paired $t$-test was used where appropriate. Associations between the study variables were assessed using the Pearson's correlation coefficient. A $p$-value of $<0.05$ was interpreted as statistically significant. Statistical analyses were performed using SAS statistical program package (SAS Institute, Cary, NC, USA) and Matlab (The MathWorks, Inc., Natick, MA, USA).

\section{RESULTS}

\section{SUBJECT CHARACTERISTICS}

The analysis is based on the data from 42 diabetic patients. The baseline characteristics of the study subjects are listed in Tables 1 and 2 . There were 13 smokers in the study group ( 7 and 6 in pravastatin and control groups, respectively). Compliance determined by capsule count exceeded 90\%. The study subjects reported no side effects during pravastatin treatment. In one subject receiving pravastatin an asymptomatic rise in serum creatine kinase level was detected at the follow-up visit. The level returned to normal within 2 weeks after cessation of medication. As a marker of glycemic control $\mathrm{GHbA}_{1 \mathrm{c}}$ levels remained unchanged during the intervention in pravastatin and placebo groups.

\section{EFFECT OF PRAVASTATIN TREATMENT ON CIRCULATING NUCLEOTIDE LEVELS AND SOLUBLE NUCLEOTIDASE ACTIVITIES}

As shown in Figure 1, there were no significant differences in ATP (panel A) and ADP (panel B) concentrations between pravastatin and placebo groups before intervention. Noteworthy, both nucleotides were maintained at significantly higher levels in plasma from diabetic patients, as compared to healthy control group.

After intervention, ATP and ADP levels decreased significantly in pravastatin group compared to pre-intervention ( $p=0.002$ and $p=0.007$, respectively), but not in the placebo group ( $p=0.06$ and $p=0.14$, respectively). Post-intervention, the ATP and ADP levels were also significantly lower in pravastatin group compared to the placebo group $(p=0.02$ and $p=0.03$, respectively), while there was no difference during preintervention ( $p=0.55$ and $p=0.50$, respectively). No signs of haemolysis or differences in plasma hemoglobin were detected among the studied groups.

Since extracellular nucleotide levels generally represent a net balance between nucleotide release and inactivation, we also

Table 1 | Baseline characteristics of the study subjects and healthy control subjects.

\begin{tabular}{llll}
\hline & $\begin{array}{l}\text { Pravastatin } \\
\text { group }\end{array}$ & $\begin{array}{l}\text { Placebo } \\
\text { group }\end{array}$ & $\begin{array}{l}\text { Healthy } \\
\text { controls }\end{array}$ \\
\hline $\mathrm{N}$ & 22 & 20 & 41 \\
Age (years) & $30.2 \pm 5.6$ & $28.9 \pm 6.5$ & $24 \pm 2.3$ \\
Males/females & $13 / 9$ & $11 / 9$ & $41 / 0$ \\
Diabetes duration (years) & $13.2 \pm 7.8$ & $10.5 \pm 5.3$ & 0 \\
BMI (kg/m ${ }^{2}$ ) & $24.7 \pm 2.5$ & $24.6 \pm 2.6$ & $24.2 \pm 2.8$ \\
FMD (\%) & $3.4 \pm 3.1$ & $4.9 \pm 3.1$ & $\mathrm{~N} / \mathrm{A}$ \\
Family history of CAD & $4 / 22$ & $2 / 20$ & $\mathrm{~N} / \mathrm{A}$ \\
Current smokers & $7 / 22$ & $6 / 20$ & $0 / 41$ \\
Background retinopathy & $5 / 22$ & $3 / 20$ & $\mathrm{~N} / \mathrm{A}$ \\
Autonomic neuropathy & $1 / 22$ & $2 / 20$ & $\mathrm{~N} / \mathrm{A}$ \\
U-Albumin/creatinine & $3 / 22$ & $0 / 20$ & $\mathrm{~N} / \mathrm{A}$ \\
male $>$ 2.5 mg/mmol & & & \\
female $>3.5$ mg/mmol & & & \\
\hline
\end{tabular}

$F M D$, flow-mediated vasodilation of brachial artery; $C A D$, coronary artery disease; N/A, data not available. 
Table 2 | Glycemic control and lipid profile of the study subjects before and after treatment, and healthy control subjects.

\begin{tabular}{|c|c|c|c|c|c|}
\hline & \multicolumn{2}{|c|}{ Pravastatin $(n=22)$} & \multicolumn{2}{|c|}{ Placebo $(n=20)$} & $\begin{array}{l}\text { Healthy control } \\
(n=41)\end{array}$ \\
\hline $\mathrm{HbA}_{1 \mathrm{c}}(\%)$ & $8.3 \pm 1.1$ & $8.4 \pm 1.2$ & $8.2 \pm 1.1$ & $8.0 \pm 1.1$ & N/A* \\
\hline Total cholesterol (mmol/L) & $4.4 \pm 0.5$ & $3.3 \pm 0.4^{* *}$ & $4.6 \pm 0.8$ & $4.3 \pm 0.6$ & $4.2 \pm 0.7$ \\
\hline LDL cholesterol (mmol/L) & $2.4 \pm 0.5$ & $1.6 \pm 0.4^{* *}$ & $2.5 \pm 0.6$ & $2.4 \pm 0.6$ & $2.3 \pm 0.6$ \\
\hline HDL cholesterol (mmol/L) & $1.6 \pm 0.3$ & $1.5 \pm 0.3$ & $1.5 \pm 0.3$ & $1.5 \pm 0.3$ & $1.5 \pm 0.4$ \\
\hline
\end{tabular}

$N / A=$ data not available. ${ }^{*}$ Reference range in non-diabetic subjects $4.2-6.0 \% ; * * p 0.001$.

A

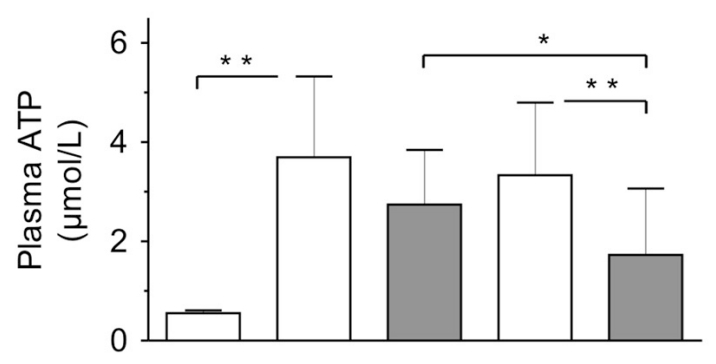

B

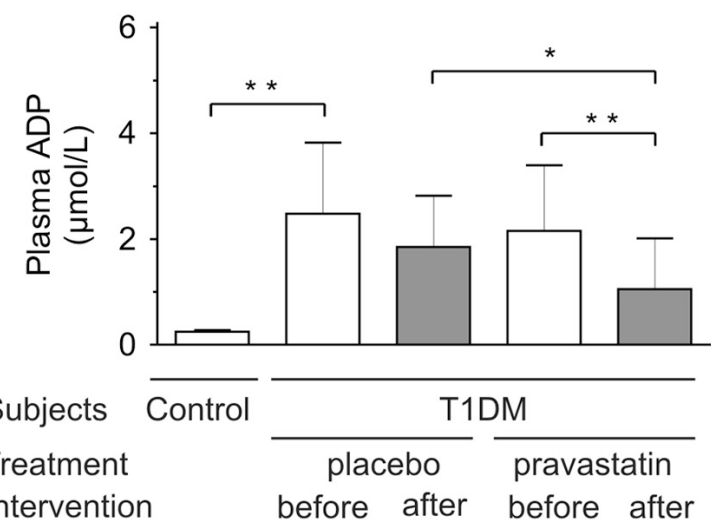

C

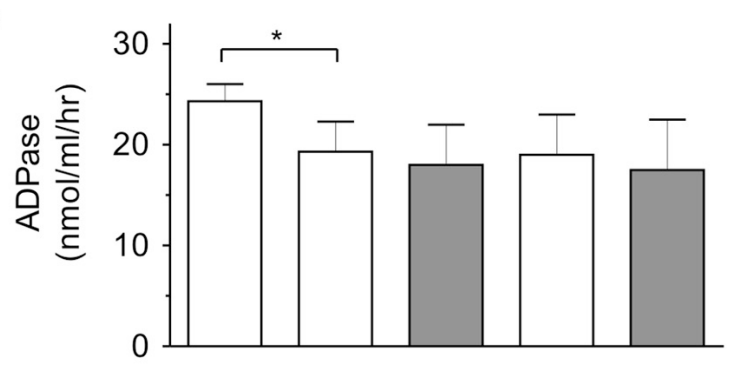

D

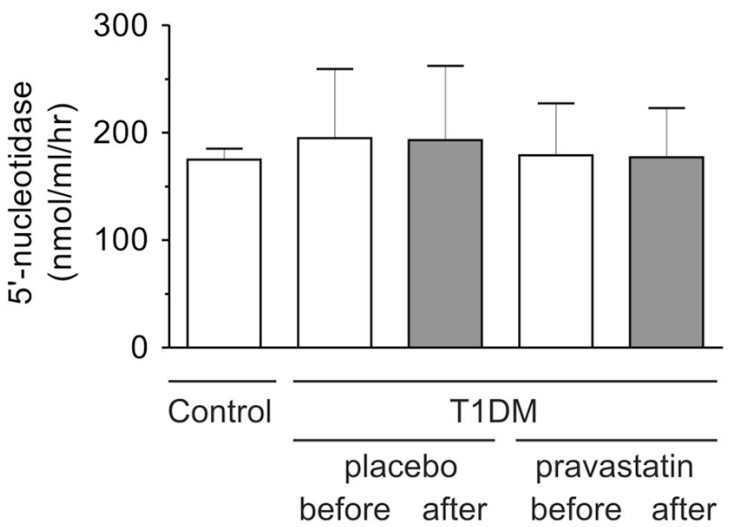

FIGURE 1 | Effect of pravastatin treatment on circulating nucleotide ATP (A) and ADP (B) levels and soluble nucleotidase ADPase (C) and $5^{\prime}$-nucleotidase activities (D) in type 1 diabetic patients (pravastatin $\boldsymbol{n}=\mathbf{2 2}$, placebo $\boldsymbol{n}=\mathbf{2 0}$ ). Control refers to healthy subjects $(n=41)$. ${ }^{*} p<0.05{ }^{* * *} p<0.01$.

determined, whether the activities of purinergic enzymes are concurrently shifted in the bloodstream of the diabetic patients. Since correct determination of serum ATPase is complicated by co-existence of two different enzymes possessing nucleotide hydrolase and pyrophosphatase activities (Yegutkin et al., 2007), soluble NTPDase was assayed in the presence of specific adenylate kinase inhibitor $\mathrm{Ap}_{5} \mathrm{~A}$ by using $\left[{ }^{3} \mathrm{H}\right] \mathrm{ADP}$ as another preferred substrate. The results unambiguously showed that the activities of serum NTPDase (Figure 1C) as well as another nucleotide-inactivating enzyme $5^{\prime}$-nucleotidase (Figure 1D) did not change in either group. Interestingly, the rate of $\left[{ }^{3} \mathrm{H}\right] \mathrm{ADP}$, but not $\left[{ }^{3} \mathrm{H}\right]$ AMP, hydrolysis by serum from diabetic patients was significantly lower in comparison with control group (Figures 1C,D).

\section{CORONARY FLOW VELOCITY RESPONSE TO COLD PRESSOR TEST}

Adequate coronary flow velocity profiles were available for 14 and 17 patients in pravastatin and placebo groups, respectively. Baseline flow velocity, hyperemia/baseline-ratio of flow velocity during CPT and time to the peak flow velocity during CPT are presented in Table 3. The acceleration to the peak flow velocity value increased in the pravastatin group from pre- to postintervention ( $p=0.02)$, but not in the placebo group $(p=0.15)$. It was also marginally different $(p=0.05)$ between the pravastatin and placebo groups post-intervention, but not at baseline $(p=0.9)$.

Baseline flow velocity and the acceleration to the peak flow velocity values were associated with post-intervention ATP levels but hyperemia to baseline ratio was not (Figure 2, panel A-C). 


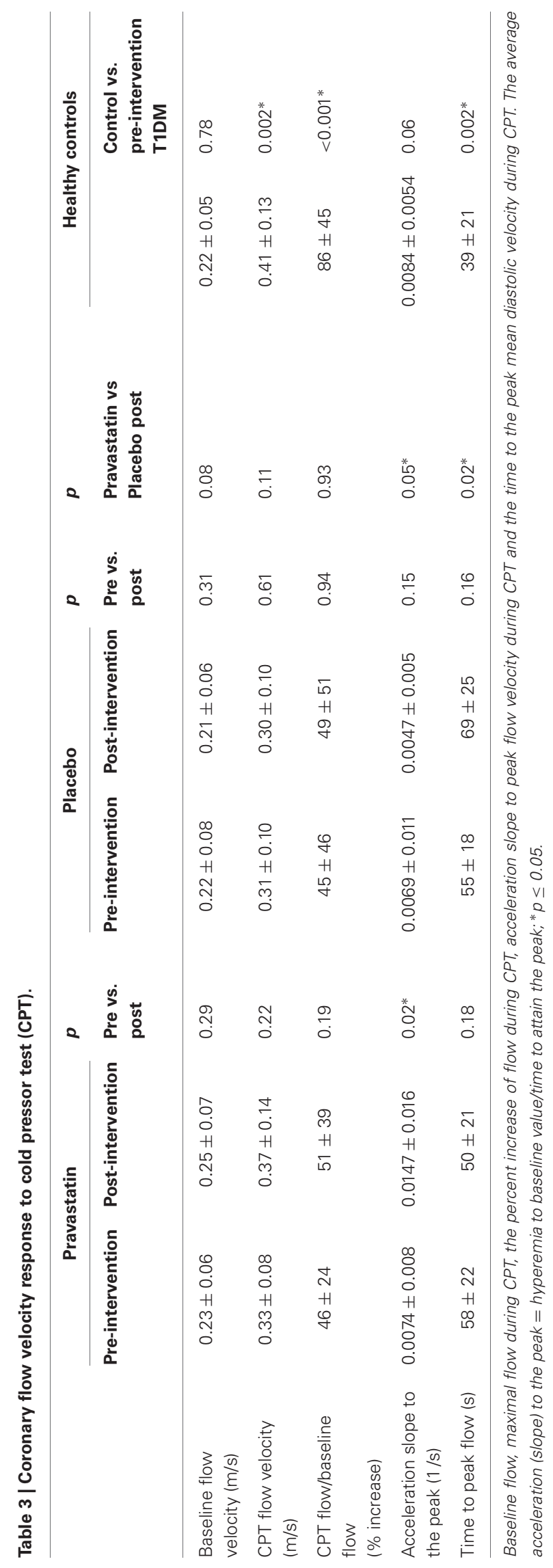



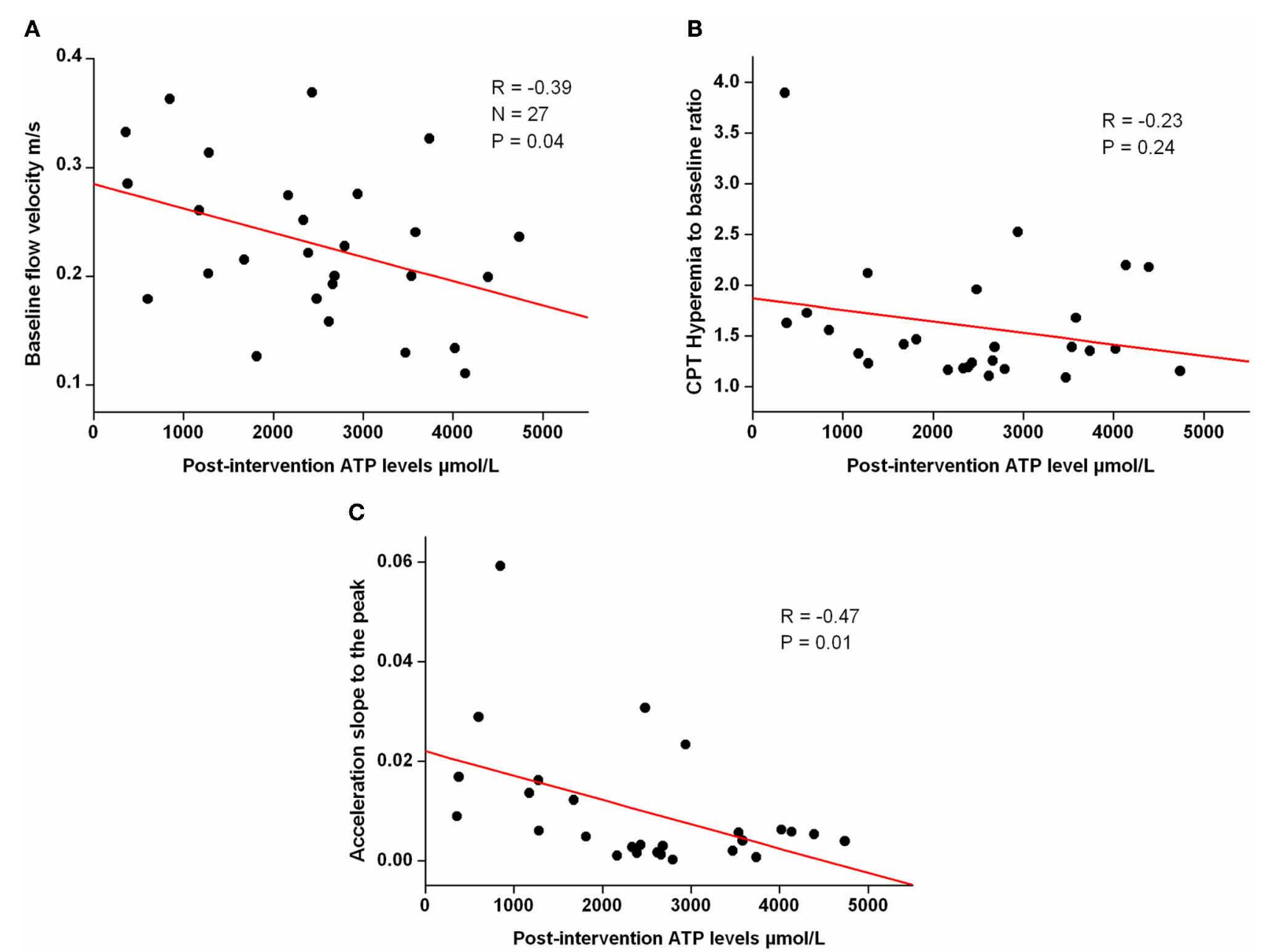

FIGURE 2 | Figure shows correlation graph of post-intervention baseline coronary flow velocity (A) $(n=27)$, CPT flow velocity/baseline flow velocity (B) $(n=26)$ and acceleration to the peak slope (C) $(n=26)$ vs. post-intervention ATP value.

No association was seen with corresponding values and the ADP levels.

\section{DISCUSSION}

The novel findings of this double blinded prospective controlled trial in patients with T1DM are that, in comparison with placebo group, four-month pravastatin treatment is accompanied by (1) significantly decreased levels of intravascular ATP and ADP without any shifts in soluble nucleotidase activities; and (2) slightly improved coronary flow responses to CPT. Moreover, T1DM patients had significantly higher plasma nucleotide concentrations in comparison with healthy controls. The obtained data on correlation between the elevated plasma ATP and ADP levels and impaired CPT might suggest the implication of purinergic signaling mechanisms in beneficial pleiotropic effects of statins in T1DM patients.

Striking novelty of this study is that, compared to healthy subjects, patients with T1DM had higher circulating ATP and
ADP levels at the fasting state and this increment can be partially restored after 4-month treatment with pravastatin. The exact mechanisms underlying the elevated pre-intervention nucleotide levels in T1DM patients remain unknown and might be particularly defined by enhanced ATP release from endothelial cells in response to chronic hyperglycemia (Parodi et al., 2002); increased shear stress due to disturbed blood flow (Yegutkin et al., 2000, 2007); and/or by diminished activity of vascular endothelial NTPDase/CD39 (Robson et al., 2005). In line with the altered nucleotide turnover results, we recently provided evidence of increased levels of circulating ATP and ADP as well as impaired coronary vasodilation response in young pre-atherosclerotic apolipoprotein E-deficient mice versus wild type littermates (Mercier et al., 2012). An increase in glucose from 5 to $15 \mathrm{mmol} / \mathrm{l}$ results in a marked increase in the proatherogenic nuclear factor of activated $\mathrm{T}$ cells signaling pathway in VSMC (Nilsson et al., 2006). The effect is mediated via glucose-induced release of ATP and 
UTP, which subsequently activate $\mathrm{P}_{2} \mathrm{Y}_{2}$ but also $\mathrm{P} 2 \mathrm{Y}_{6}$ receptors after degradation to UDP. Thus, nucleotide release is a potential metabolic sensor for the arterial smooth muscle response to high glucose. (Erlinge and Burnstock, 2008) In this context, it is relevant to emphasize that serum NTPDase activities were lower in diabetics, as compared to those of the healthy control group. Moreover, while no significant shifts in soluble nucleotidase activities were detected in the sera from pravastatin-treated T1DM patients versus placebo group, we do not exclude the possibility of statin-mediated up-regulation of vascular nucleotidase activities, which in turn would affect the levels of circulating nucleotides and tune their signaling responses in the cardiovascular system. This hypothesis is indirectly supported by previous in vitro data on the ability of various statins (lovastatin, atorvastatin) to increase the activities of ecto-5' -nucleotidase (Ledoux et al., 2002; Sanada et al., 2004; Osman et al., 2006) and other nucleotide-inactivating enzymes (Osman et al., 2006) in vascular endothelial and other cells. Irrespective of the underlying mechanisms, the increment of plasma ATP and ADP in T1DM patients and their decrease after pravastatin treatment would reasonably suggest that a net balance between nucleotide release and inactivation is disturbed in diabetes and it can be partially restored by statins.

This is the first study assessing the role of purinergic signaling molecules in the early stages of coronary microcirculatory dysfunction of T1DM patients without any clinical signs or symptoms of macrovascular disease or microvascular complications. Due to small sample size, the physiological responses to CPT need to be interpreted cautiously. The primary end point-hyperemia to baseline ratio-was not significantly altered, and there was only a slight statistically non-significant increase in baseline flow velocity after pravastatin treatment in T1DM patients. The co-primary end point (acceleration slope to the peak and time to peak flow) differences were more obvious though subtle between the pravastatin and placebo groups. Compared to healthy men, pre-intervention hyperemic flow response was lower in T1DM patients in terms of lower peak flow velocity and longer acceleration time to peak.

We intentionally selected subjects with normal cholesterol levels and good to satisfactory glycemic control who are likely to be representative of the majority of young patients with type 1 diabetes not generally receiving lipid-lowering therapy although being in clearly increased risk in developing CAD. At the time of the recruitment the study subjects had no diagnoses of diabetic complications other than background retinopathy and microalbuminuria in a small number of the subjects. Autonomic neuropathy in type 1 diabetes has been suggested to cause hyper-reactivity to nitro vasodilators in the forearm vasculature (Mäkimattila et al., 1997). Therefore we did not include subjects with FMD of the brachial artery more than $10 \%$ in the study. Pravastatin treatment was generally well tolerated and did not worsen glycemic control. Despite the physiological variability of enzymatic activities/nucleotide concentrations for each individual was relatively high (within 18-47\%), there was a distinct difference in ATP and ADP levels between T1DM patients and the control group pre-intervention. Moreover, the decrease in ATP levels after intervention was greater than the physiological variability.

Some limitations need to be addressed. Firstly, caffeine-a competitive adenosine receptor antagonist—was withdrawn $12 \mathrm{~h}$ before blood sample collection, and CPT and FMD tests. It is possible that some of its effect persisted at the time of testing. Secondly, we measured soluble ADPase and 5' -nucleotidase activities, but also other methods of nucleotidase activity assessment have been reported. In a recent report, the effect of rosuvastatin treatment on ecto-5'-nucleotidase activity on the surface of human mononuclear cells was measured and its up-regulation further correlated with vasodilatory responses to ischemia (Meijer et al., 2010). Nevertheless, this rather heterogenous cell population represents only certain fraction of circulating hematopoietic cells and express relatively low ecto5 -nucleotidase activity as compared to vascular endothelial cells (Yegutkin et al., 2002), with the latter cells being generally considered the major regulators of intravascular purinergic signaling and hyperaemic responses. From this point of view, we believe that soluble serum $5^{\prime}$-nucleotidase-in conjunction with another key inactivating enzyme NTPDase1/CD39-represents more integrative parameter of systemic shifts in the enzyme levels and might open up further research to assess the potential diagnostic application of purine-converting enzymes in clinical biochemistry.

\section{CONCLUSIONS}

Intravascular nucleotide turnover is altered in the early stages of atherosclerosis in T1DM patients as compared to healthy control subjects. Four-month treatment with pravastatin in young normocholesterolemic patients with non-complicated type 1 diabetes decreased circulating ATP and ADP levels and concurrently improved coronary microvascular reactivity to $\mathrm{CPT}$ as compared to placebo. The existence of correlation between intravascular ATP and ADP and coronary reactivity also indicates on the physiological relevance of the measured systemic nucleotide levels and may open up further research for future therapeutic or diagnostic applications of purinergic signaling pathways in the treatment of diabetes and other diseases.

\section{ACKNOWLEDGMENTS}

The study is part of the project "Molecular Imaging in Cardiovascular and Metabolic Research," which belongs to the Centre of Excellence program of the Academy of Finland. The study was also supported by grants from the Sigrid Juselius Foundation, the Finnish Cultural Foundation, Finnish Cardiovascular Foundation, and the Academy of Finland (grants no. 201888, 120569, and 74092). Bristol-Myers Squibb Finland provided the investigational drugs free of charge. 


\section{REFERENCES}

Bours, M. J. L., Swennen, E. L. R., Di Virgilio, F., Cronstein, B. N., and Dagnelie, P. C. (2006). Adenosine $5^{\prime}$-triphosphate and adenosine as endogenous signaling molecules in immunity and inflammation. Pharmacol. Ther. 112, 358-404.

Dimitrow, P. P., Krzanowski, M., Nizankowski, R., Szczeklik, A., and Dubiel, J. S. (2000). Comparison of the effect of verapamil and propranolol on response of coronary vasomotion to cold pressor test in symptomatic patients with hypertrophic cardiomyopathy. Cardiovasc. Drug Ther. 14, 643-650.

Eltzschig, H. K., Ibla, J. C., Furuta, G. T., Leonard, M. O., Jacobson, K. A., Enjyoji, K., Robson, S. C., and Colgan, S. P. (2003). Coordinated adenine nucleotide phosphohydrolysis and nucleoside signaling in posthypoxic endothelium. J. Exp. Med. 198, 783-796.

Erlinge, D., and Burnstock, G. (2008). $\mathrm{P} 2$ receptors in cardiovascular regulation and disease. Purinergic Signal. 4, 1-20.

Janatuinen, T., Knuuti, J., Toikka, J., Ahotupa, M., Nuutila, P., Rönnemaa, T., and Raitakari, O. (2004). Effect of pravastatin on low-density lipoprotein oxidation and myocardial perfusion in young adults with type 1 diabetes. Arterioscler. Thromb. Vasc. Biol. 24, 1303-1308.

Järvisalo, M. J., Raitakari, M., Toikka, J. O., Putto-Laurila, A., Rontu, R., Laine, S., Lehtimäki, T., Rönnemaa, T., Viikari, J., and Raitakari, O. T. (2004). Endothelial dysfunction and increased arterial intimamedia thickness in children with type 1 diabetes. Circulation 109, 1750-1755.

Kiviniemi, T. O., Toikka, J. O., Koskenvuo, J. W., Saraste, A., Saraste, M., Pärkkä, J. P., Raitakari, O. T., and Hartiala, J. J. (2007). Vasodilation of epicardial coronary artery can be measured with transthoracic echocardiography. Ultrasound Med. Biol. 33, 362-370.

Kiviniemi, T. (2008). Assessment of coronary blood flow and the reactivity of the microcirculation non-invasively with transthoracic echocardiography. Clin. Physiol. Funct. Imaging 28, 145-155.

Laufs, U., La Fata, V., Plutzky, J., and Liao, J. (1998). Upregulation of endothelial nitric oxide synthase by $\mathrm{HMG}$ CoA reductase inhibitors. Circulation 97, 1129-1135.

Ledoux, S., Laouari, D., Essig, M., Runembert, I., Trugnan, G., Michel, J. B., and Friedlander, G. (2002). Lovastatin enhances ecto5 -nucleotidase activity and cell surface expression in endothelial cells: implication of Rho-family GTPases. Circ. Res. 90, 420-427.

Mäkimattila, S., Mäntysaari, M., Groop, P., Summanen, P., Virkamäki, A., Schlenzka, A., Fagerudd, J., and Yki-Järvinen, H. (1997). Hyperreactivity to nitrovasodilators in forearm vasculature is related to autonomic dysfunction in insulin-dependent diabetes mellitus. Circulation 95, 618-625.

Meijer, P., Wouters, C. W., van den Broek, P. H. H., de Rooij, M., Scheffer, G. J., Smits P, and Rongen, G. A. (2010). Upregulation of ecto$5^{\prime}$-nucleotidase by rosuvastatin increases the vasodilator response to ischemia. Hypertension 56, 722-727.

Mercier, N., Kiviniemi, T. O., Saraste, A., Miiluniemi, M., Silvola, J., Jalkanen, S., and Yegutkin, G. G. (2012). Impaired ATP-induced coronary blood flow and diminished aortic NTPDase activity precede lesion formation in apolipoprotein E-Deficient mice. Am. J. Pathol. 180, 419-428.

Merla, R., Daher, I. N., Ye, Y., Uretsky, B. F., and Birnbaum, Y. (2007). Pretreatment with statins may reduce cardiovascular morbidity and mortality after elective surgery and percutaneous coronary intervention: clinical evidence and possible underlying mechanisms. Am. Heart J. 154, 391-402.

Nilsson, J., Nilsson, L. M., Chen, Y., Molkentin, J. D., Erlinge, D, and Gomez, M. F. (2006). High glucose activates nuclear factor of activated $\mathrm{T}$ cells in native vascular smooth muscle. Arterioscler. Thromb. Vasc. Biol. 26, 794-800.

Osman, L., Chester, A. H., Amrani, M., Yacoub, M. H., and Smolenski, R. T. (2006). A novel role of extracellular nucleotides in valve calcification: a potential target for atorvastatin. Circulation 114, I566-I572.

Parodi, J., Flores, C., Aguayo, C., Rudolph, M. I., Casanello, P., and Sobrevia, L. (2002). Inhibition of nitrobenzylthioinosine-sensitive adenosine transport by elevated $\mathrm{D}$ glucose involves activation of $\mathrm{P} 2 \mathrm{Y} 2$ purinoceptors in human umbilical vein endothelial cells. Circ. Res. 90, 570-577.

Pitkänen, O. P., Nuutila, P., Raitakari, O. T., Rönnemaa, T., Koskinen, P. J., Iida, H., Lehtimäki, T. J., Laine, H. K., Takala, T., Viikari, J. S., and Knuuti, J. (1998). Coronary flow reserve is reduced in young men with IDDM. Diabetes 47, 248-254.

Ralevic, V., and Burnstock, G. (1998). Receptors for purines and pyrimidines. Pharmacol. Rev. 50, 413-492.

Robson, S. C., Wu, Y., Sun, X., Knosalla, C., Dwyer, K., and Enjyoji, K. (2005). Ectonucleotidases of CD39 family modulate vascular inflammation and thrombosis in transplantation. Semin. Thromb. Hemost. 31, 217-233.

Sanada, S., Asanuma, H., Minamino, T., Node, K., Takashima, S., Okuda, H., Shinozaki, Y., Ogai, A., Fujita, M., Hirata, A., Kim, J., Asano, Y., Mori, H., Tomoike, H., Kitamura, S., Hori, M., and Kitakaze, M. (2004). Optimal windows of statin use for immediate infarct limitation: $5^{\prime}$-nucleotidase as another downstream molecule of phosphatidylinositol 3-kinase. Circulation 110, 2143-2149.

Yegutkin, G. G., and Burnstock, G. (1998). Steady-state binding of $[3 \mathrm{H}]$ ATP to rat liver plasma membranes and competition by various purinergic agonists and antagonists. Biochim. Biophys. Acta 1373, 227-236.

Yegutkin, G. G., Henttinen, T., Samburski, S. S., Spychala, J., and Jalkanen, S. (2002). The evidence for two opposite, ATP-generating and ATP-consuming, extracellular pathways on endothelial and lymphoid cells. Biochem. J. 367, 121-128.

Yegutkin, G. G., Samburski, S. S., Mortensen, S. P., Jalkanen, S. and González-Alonso, J. (2007). Intravascular ADP and soluble nucleotidases contribute to acute prothrombotic state during vigorous exercise in humans. J. Physiol. 579, 553-564.

Yegutkin, G., Bodin, P., and Burnstock, G. (2000). Effect of shear stress on the release of soluble ecto-enzymes ATPase and 5?-nucleotidase along with endogenous ATP from vascular endothelia cells. Br. J. Pharmacol. 129, 921-926.

Conflict of Interest Statement: The authors declare that the research was conducted in the absence of any commercial or financial relationships that could be construed as a potential conflict of interest.

Received: 05 June 2012; accepted: 01 August 2012; published online: 23 August 2012.

Citation: Kiviniemi TO, Yegutkin GG, Toikka JO, Paul S, Aittokallio T, Janatuinen T, Knuuti J, Rönnemaa T, Koskenvuo JW, Hartiala JJ, Jalkanen S and Raitakari OT (2012) Pravastatininduced improvement in coronary reactivity and circulating ATP and ADP levels in young adults with type 1 diabetes. Front. Physio. 3:338. doi: 10.3389/fphys.2012.00338

This article was submitted to Frontiers in Clinical and Translational Physiology, a specialty of Frontiers in Physiology.

Copyright (C) 2012 Kiviniemi, Yegutkin, Toikka, Paul, Aittokallio, Janatuinen, Knuuti, Rönnemaa, Koskenvuo, Hartiala, Jalkanen and Raitakari. This is an open-access article distributed under the terms of the Creative Commons Attribution License, which permits use, distribution and reproduction in other forums, provided the original authors and source are credited and subject to any copyright notices concerning any third-party graphics etc. 